\title{
Kasuistiken
}

Ophthalmologe 2022 $\cdot 119: 395-399$ https://doi.org/10.1007/s00347-021-01367-8 Eingegangen: 2. Februar 2021

Angenommen: 28. Februar 2021

Online publiziert: 29. März 2021

c c Der/die Autor(en) 2021

\author{
Christian S. Mayer ${ }^{1}$ (D) $\cdot$ Isabella D. Baur ${ }^{1} \cdot J^{\prime}$ lia Storr ${ }^{2} \cdot$ Ramin Khoramnia $^{1}$ \\ ${ }^{1}$ Augenklinik, Universitätsklinikum Heidelberg, Heidelberg, Deutschland \\ ${ }^{2}$ Klinik und Poliklinik für Augenheilkunde, Klinikum rechts der Isar, München, Deutschland
}

\section{Traumatische Aniridie: Konservativer oder chirurgischer Therapieansatz?}

re Argonlaser-Koagulationsherde bei ansonsten regelrechtem Befund, jedoch bei reduziertem Einblick aufgrund der Katarakt. Eine Lentodonesis konnte nicht festgestellt werden. Am rechten Auge zeigten sich ein reizfreier Vorderabschnitt mit altersentsprechend klarer Linse sowie ein fundoskopischer Normalbefund. Der intraokulare Druck lag beidseits im Normbereich. Am linken Auge war die Endothelzellzahl mit 1803 Zellen/mm² verringert, wohingegen die Endothelzellzahl am rechten Auge mit 3039 Zellen/mm² normwertig war. Die Kontrastsensitivität wurde mit der Pelli-Robson-Tafel gemessen und ergab für das linke Auge einen Wert von 0,45 „log units“. Der Patient wurde gebeten, die subjektive kosmetische Beeinträchtigung und die subjektive Beeinträchtigung durch Blendung auf einer Skala von 1 bis $10 \mathrm{zu}$ bewerten, wobei 1 für eine geringe Beeinträchtigung und 10 für eine sehr starke Beeinträchtigung steht. Die subjektive Beeinträchtigung durch Blendung wurde vom Patienten mit 2 bewertet, die subjektive kosmetische Beeinträchtigung wurde mit 6 bewertet. Die $\bullet$ Abb. 1a, b zeigt den präoperativen Befund ohne und • Abb. 1c, d mit Irisprintkontaktlinse.

\section{Therapie und Verlauf}

Der Patient wurde darüber aufgeklärt, dass durch eine Kataraktoperation am linken Auge zwar ein Visusanstieg möglich ist, jedoch die Prognose aufgrund von möglichen (subklinischen) Veränderungen der Netzhaut eingeschränkt ist. Die Möglichkeit einer kombinierten Implantation einer Intraokularlinse (IOL) mit einer Artificial-Iris (AI) wurde mit dem Patienten und den Eltern diskutiert - dies insbesondere im Hinblick auf die Möglichkeit, auf die Iriskontaktlinse zu verzichten, mit derzeitigen Mitteln guten funktionellen und ästhetischen Ergebnissen sowie dem gegenüber einer zweizeitigen Operation geringeren Operationsrisiko. Über die möglichen Komplikationen inklusive einer Hornhautdekompensation bis hin zur Notwendigkeit einer Keratoplastik oder der Entwicklung eines Glaukoms wurde der Patient ausführlich aufgeklärt. Bei entsprechendem Leidensdruck entschied sich der Patient für den vorgeschlagenen Eingriff.

Am linken Auge erfolgte die Implantation einer Intraokularlinse (Aspira-AaY +17,0 dpt., Human Optics, Erlangen, Deutschland), eines Kapselspannringes und der zuvor individuell angefertigten AI (Customflex ArtificialIris, Human Optics, Erlangen, Deutschland) in den Kapselsack (In-the-bag-Technik) in einem Eingriff (• Abb. 2).

Fünf Monate postoperativ konnten wir am linken Auge einen Anstieg des bestkorrigierten Visus auf 0,32 dezimal mit einer subjektiven Refraktion von $+1,5 /-1,5 / 170^{\circ}$ feststellen. Die Endothelzellzahl war mit 1753 Zellen/mm² stabil, und der intraokulare Druck lag weiterhin im Normbereich. Die Kontrastsensitivität, gemessen mit der PelliRobson-Tafel, stieg auf 1,35 „log units“ an. Postoperativ bewertete der Patient die subjektive Blendung unverändert mit 2, die kosmetische Beeinträchtigung wurde nun mit dem niedrigsten möglichen Wert von 1 bewertet. Postoperativ wurde der Patient gebeten, auch seine 


\section{Kasuistiken}
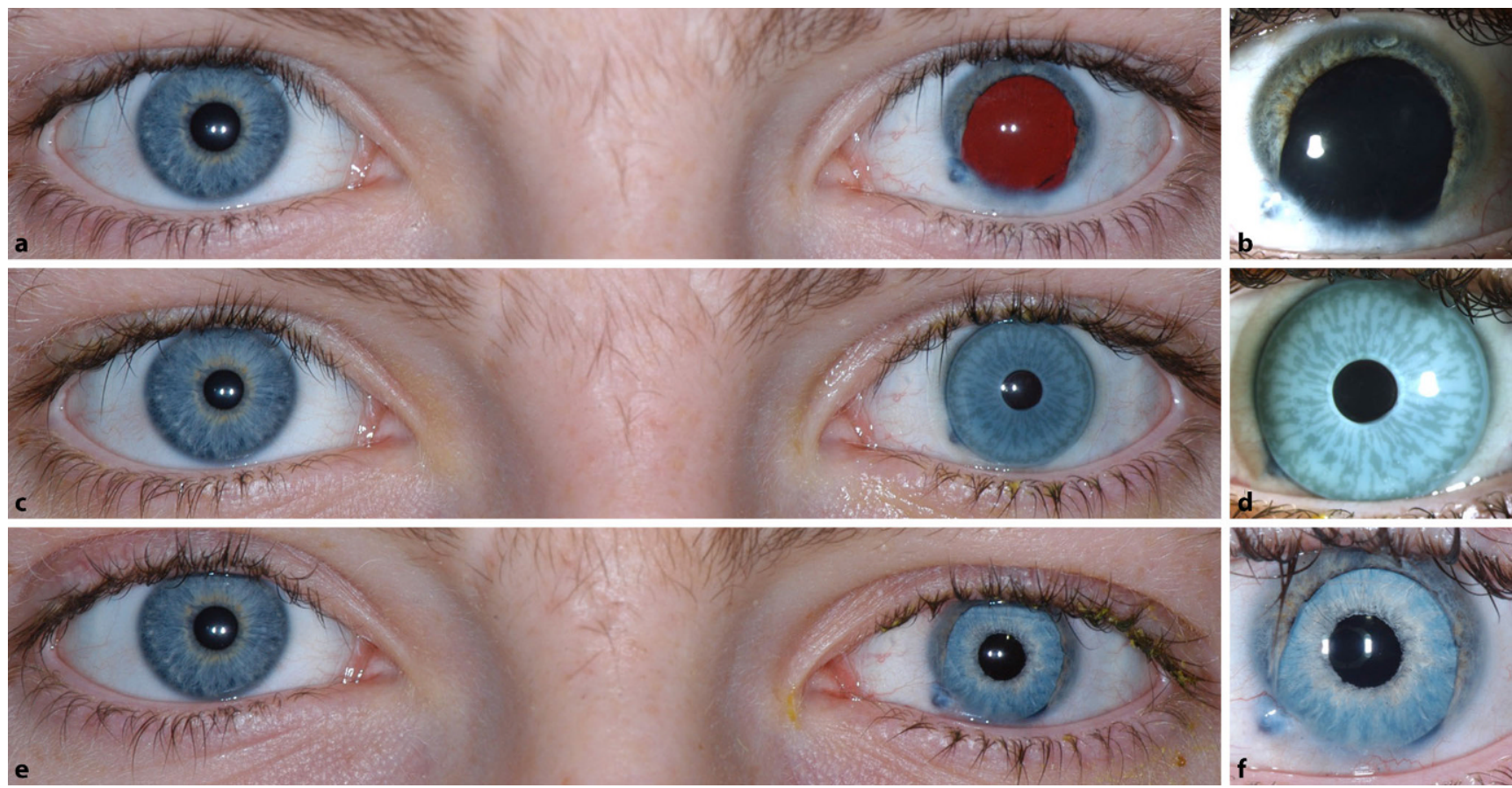

Abb. $1 \Delta$ a, b Präoperativer Befund. Der ausgedehnte Irisdefekt von 5-7 h ist deutlich zu erkennen. c, d Versorgung mit einer Irisprintkontaktlinse. e, f Postoperatives Ergebnis nach Versorgung mit einer Intraokularlinse und Artificial-Iris

Zufriedenheit mit dem Ergebnis auf einer Skala von 1 bis 10 zu bewerten, wobei 1 für geringe und 10 für sehr hohe $\mathrm{Zu}$ friedenheit steht. Der Patient gab hierfür den Wert 9 an. Die - Abb. 1e, $\mathbf{f}$ zeigt das postoperative Ergebnis. - Abb. 3 zeigt eine postoperative Spectral-Domain Optische Kohärenztomographie (SD-OCT) des linken Auges.

\section{Diskussion}

Die Versorgung unseres jungen Patienten mit einer AI in Kombination mit einer Intraokularlinse verlief komplikationslos. Wir konnten ein sehr gutes ästhetisches Ergebnis und eine sehr hohe Patientenzufriedenheit beobachten. Auch einen Visusanstieg und eine Verbesserung des Kontrastsehens konnten wir verzeichnen. Obwohl das primäre Ziel bei der AI Implantation nicht die Visusverbesserung, sondern die Reduktion der Blendempfindlichkeit ist, wurde ein Visusanstieg schon mehrfach berichtet, was am ehesten auf die oftmals im gleichen Eingriff durchgeführte IOL-Implantation zurückzuführen ist [1-4]. Es wurde gezeigt, dass durch die AI-Implantation eine signifikante Reduktion des Pupillendurchmessers sowie ein Anstieg des
Kontrastsehens erreicht werden können $[1,5]$.

Die Customflex ArtificialIris wird für jeden Patienten individuell angefertigt. Dazu wird die Vorderseite des flexiblen Silikonimplantates von Hand bemalt, um es dem Aussehen des verbliebenen Irisgewebes und des Partnerauges möglichst genau anzupassen. Hierzu ist eine farbgetreue Fotodokumentation vor der Anfertigung zwingend erforderlich. Durch die individuelle Anpassung des Implantates können ausgesprochen gute kosmetische Ergebnisse erreicht werden [2, 5-7]. Gerade junge Patienten legen oftmals besonderen Wert auf das äußere Erscheinungsbild und profitieren bezüglich der subjektiven Beeinträchtigung durch die ästhetische Entstellung von einem rekonstruktiven Eingriff.

Die künstliche Iris kann mit verschiedenen Verfahren in das Auge implantiert werden [8-10]. Bei unserem Patienten konnte die AI zusammen mit einer IOL und einem Kapselspannring in den Kapselsack implantiert werden. Der Kapselspannring soll einer Verkippung der AI durch eine Fibrose und Kontraktion des Kapselsackes vorbeugen. Die Berechnung der IOL-Stärke kann mittels Biometrie ohne Korrekturfaktor erfolgen [11].
Die effektive Linsenposition wird durch die zusätzliche Implantation der künstlichen Iris nur minimal verändert, was die postoperative Refraktion bestätigte.

In manchen Anwendungsfällen wurde ein Anstieg des intraokularen Drucks nach AI-Implantation beschrieben [3, 12]. Patienten mit einem vorbestehenden Glaukom gelten als Risikogruppe für einen postoperativen Druckanstieg, wobei nicht in allen Fällen eine eindeutige Ursache wie ein Pupillarblock oder Pigmentdispersionsglaukom identifiziert werden konnte. Bislang ist noch unklar, ob Iridektomien an der künstlichen Iris einen Pupillarblock wirksam verhindern können [3, 8]. Auch eine okuläre Hypotension kann nach der AIImplantation auftreten, wobei auch hier Patienten mit einem bereits präoperativ verminderten intraokularen Druck aufgrund eines schweren okulären Traumas häufiger betroffen sind [12]. Ähnlich wie bei einer Kataraktoperation kann die Implantation einer AI zum Endothelzellverlust führen $[3,12,13]$, jedoch kann dies bei einer bereits durch ein Trauma reduzierten Endothelzellzahl zu einer Hornhautdekompensation bis hin zur Notwendigkeit einer Keratoplastik führen. Auch eine (Sub-)Luxation einer 

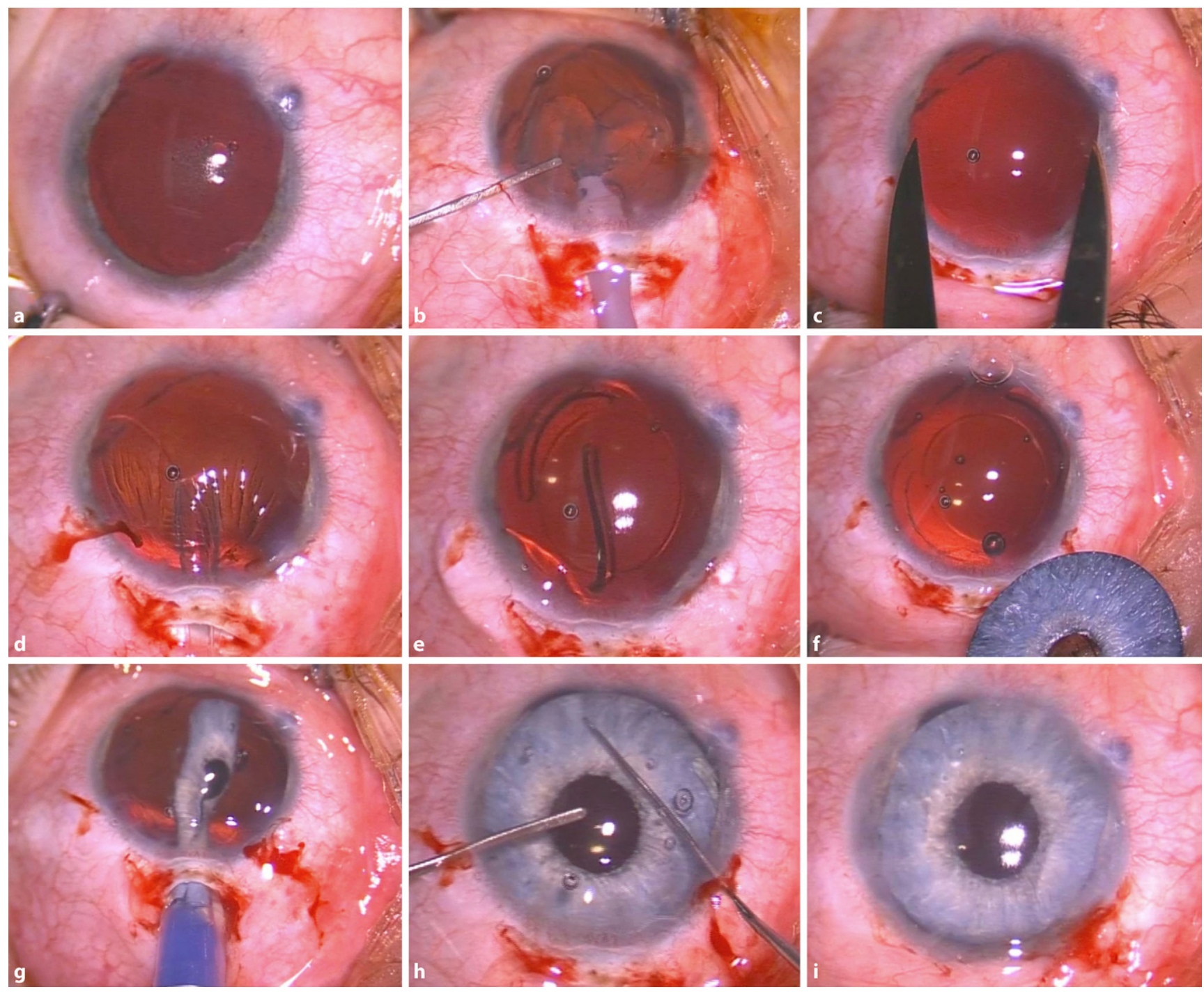

Abb. 2 \ a Präoperativer Befund. b Phakoemulsifikation durch die relativ große Rhexis über einen sklerokornealen Tunnel. cBestimmung der Trepanationsgröße für das Irisimplantat. d Implantation eines Kapselspannringes. e Implantation der Intraokularlinse.f Vorbereiten der künstlichen Iris extraokular. $\mathrm{g}$ Injektorassistierte Implantation der flexiblen gerollten Silikoniris in den Kapselsack vor die IOL. $\mathbf{h}$ Positionierung und Zentrierung sowie Absaugung des Viskoelastikums aus der Vorderkammer. i Befund am Ende der Operation mit guter Zentrierung

sulcus- oder nahtfixierten AI mit dem Risiko einer Luxation in den Glaskörperraum kann auftreten [3]. In diesen Fällen ist eine Reposition, ggf. mit Nahtfixierung der AI erforderlich, um Schäden an der Netzhaut zu verhindern $[3,12]$. Neben chronischer Inflammation und zystoidem Makulaödem gehört auch das sog. "residual iris retraction syndrome“ (RITS) zu den bekannten Komplikationen nach AI-Implantation. Beim RITS kommt es aus unbekannter Ursache zu einer progredienten Vergrößerung der natürlichen Pupillenöffnung, die mit einer chronischen Entzündungsreaktion und erhöhtem intraokularem Druck in Verbindung gebracht wird [14]. Das Risiko für Komplikationen hängt maßgeblich vom Ausmaß der Verletzungen eines Auges und der gewählten Technik für die Implantation ab. In unserem Fall mit der Möglichkeit der Implantation in den Kapselsack bei einem verhältnismäßig stabilen präoperativen Befund durften wir von einem eher geringen intra- und postoperativen Komplikationsrisiko ausgehen.

Alternative Implantate sind das Aniridieimplantat Type 68 von Morcher (Stuttgart, Deutschland), das nur in schwarzer Farbe erhältlich ist, oder das Irisimplantat Modell C1/F1 der Firma Ophtec
(Groningen, Niederlande), das in ca. 120 verschiedenen Designs hergestellt wird. Beide Implantate sind mit eingearbeiteter Optik erhältlich, haben jedoch den Nachteil, dass das ästhetische Ergebnis aufgrund der eingeschränkten Farbauswahl und fehlenden Individualisierung nicht mit dem in diesem Fall verwendeten Implantat vergleichbar ist. Außerdem ist es nicht möglich, diese in den Kapselsack zu implantieren.

Zusammenfassend stellt die Implantation einer AI zusammen mit einer Intraokularlinse und einem Kapselspannring in den Kapselsack eine elegante Methode dar, um bei einer traumatischen Aniri- 


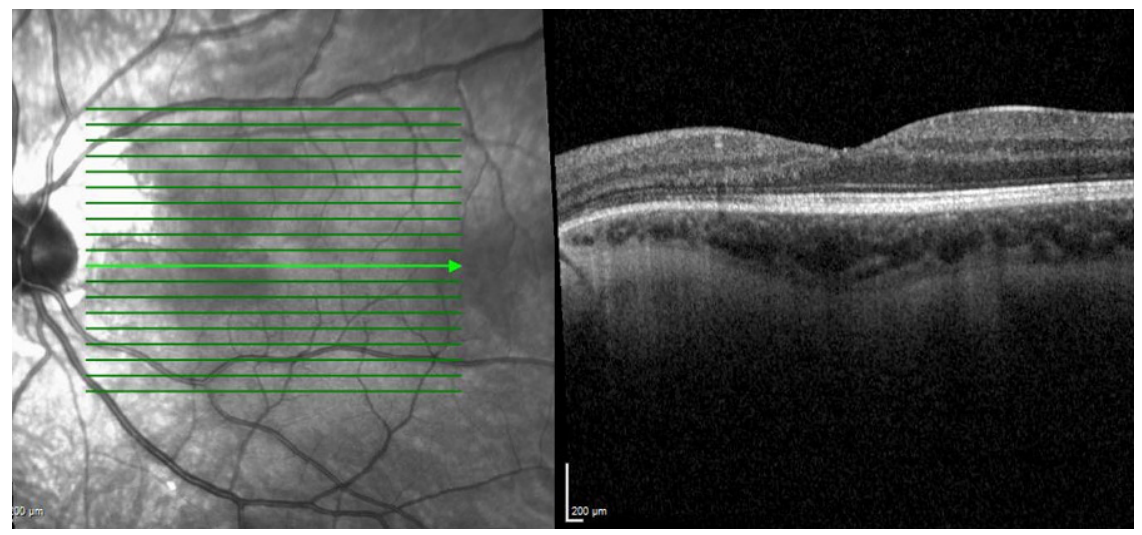

Abb. 3 ॥ Spectral-Domain Optische Kohärenztomographie (SD-OCT) des linken Auges mit Residualzustand nach stumpfem Bulbustrauma: intraretinale Strukturveränderungen sowie Defekte der Photorezeptorschicht im papillomakulären Bündel

die und Katarakt Funktion und Ästhetik auch bei sehr jungen Patienten in einer einzigen Operation wiederherzustellen. Gerade bei jungen Patienten muss auch das langfristige Komplikationsrisiko berücksichtigt werden, da das Implantat noch viele Jahre im Auge verbleiben wird.

\section{Fazit}

1. Auf den ersten Blick stellt die Irisprintkontaktlinse eine gute Therapieoption bei einer traumatischen Aniridie dar. Jedoch ist die Anwendung komplex und teuer.

2. Selbst bei vermeintlich guter Optik bei der Aufsicht (- Abb. 1c, d) können Patienten über Unzufriedenheit mit Irisprintkontaktlinsen klagen. Die Farbe befindet sich auf der gekrümmten Oberfläche der Kontaktlinse. Dies wirkt insbesondere beim Blick von der Seite auf das Auge des Patienten unnatürlich.

3. Im Falle einer zusätzlich bestehenden traumatischen Katarakt ist eine alleinige Versorgung mit Irisprintkontaktlinsen nicht mehr zielführend.

4. Im Rahmen einer ohnehin durchzuführenden Kataraktoperation kann mit vergleichsweise geringem Aufwand auch die Aniridie behandelt werden. Die Operation ist insbesondere dann risikoarm, wenn die künstliche Iris und die IOL gemeinsam in den Kapselsack implantiert werden können.

\section{Korrespondenzadresse

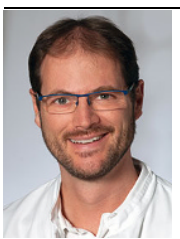 \\ PD Dr. med. \\ Christian S. Mayer \\ Augenklinik, Universitätsklini- \\ kum Heidelberg \\ Im Neuenheimer Feld 400, \\ 69120 Heidelberg, \\ Deutschland \\ Christian.Mayer@med.uni- \\ heidelberg.de}

Funding. Open Access funding enabled and organized by Projekt DEAL.

\section{Einhaltung ethischer Richtlinien}

Interessenkonflikt. C.S. Mayer gibt an, während der Studiendurchführung persönliche Honorare und nichtfinanzielle Unterstützung von HumanOptics erhalten zu haben. I.D. Baur gibt an, unabhängig von der eingereichten Arbeit nichtfinanzielle Unterstützung von Santen erhalten zu haben. J. Storr gibt an, dass kein Interessenkonflikt besteht. R. Khoramnia gibt an, unabhängig von der eingereichten Arbeit folgende Zuwendungen erhalten zu haben: Forschungsgelder, persönliche Honorare und nichtfinanzielle Unterstützung von Alcon, Forschungsgelder von Zeiss, Forschungsgelder und persönliche Honorare von PhysIOL, Forschungsgelder, persönliche Honorare und nichtfinanzielle Unterstützung von Johnson \& Johnson, Forschungsgelder, persönliche Honorare und nichtfinanzielle Unterstützung von Santen, Forschungsgelder, persönliche Honorare und nichtfinanzielle Unterstützung von Kowa, Forschungsgelder, persönliche Honorare und nichtfinanzielle Unterstützung von Hoya, Forschungsgelder und persönliche Honorare von Ophtec, Forschungsgelder, persönliche Honorare und nichtfinanzielle Unterstützung von Rayner, Forschungsgelder, persönliche Honorare und nichtfinanzielle Unterstützung von SIFI, Forschungsgelder und persönliche Honorare von Acufocus, Forschungsgelder, persönliche Honorare und nichtfinanzielle Unterstützung von Oculentis.
Für diesen Beitrag wurden von den Autoren keine Studien an Menschen oder Tieren durchgeführt. Für die aufgeführten Studien gelten die jeweils dort angegebenen ethischen Richtlinien. Für Bildmaterial oder anderweitige Angaben innerhalb des Manuskripts, über die Patienten zu identifizieren sind, liegt von innen und/oder ihren gesetzlichen Vertretern eine schriftliche Einwilligung vor.

Open Access. Dieser Artikel wird unter der Creative Commons Namensnennung 4.0 International Lizenz veröffentlicht, welche die Nutzung, Vervielfältigung, Bearbeitung, Verbreitung und Wiedergabe in jeglichem Medium und Format erlaubt, sofern Sie den/die ursprünglichen Autor(en) und die Quelle ordnungsgemäß nennen, einen Link zur Creative Commons Lizenz beifügen und angeben, ob Änderungen vorgenommen wurden.

Die in diesem Artikel enthaltenen Bilder und sonstiges Drittmaterial unterliegen ebenfalls der genannten Creative Commons Lizenz, sofern sich aus der Abbildungslegende nichts anderes ergibt. Sofern das betreffende Material nicht unter der genannten Creative Commons Lizenz steht und die betreffende Handlung nicht nach gesetzlichen Vorschriften erlaubt ist, ist für die oben aufgeführten Weiterverwendungen des Materials die Einwilligung des jeweiligen Rechteinhabers einzuholen.

Weitere Details zur Lizenz entnehmen Sie bitte der Lizenzinformation auf http://creativecommons.org/ licenses/by/4.0/deed.de.

\section{Literatur}

1. Mayer CS, Hoffmann AM, Prahs P et al (2020) Functional outcomes after combined iris and intraocular lens implantation in various iris and lens defects. BMCOphthalmol 20:1-7

2. Rickmann A, Szurman P, JanuschowskiKetal (2016) Long-term results after artificial iris implantation in patients with aniridia. Graefes Arch Clin Exp Ophthalmol 254:1419-1424

3. Mayer CS, Laubichler AE, Khoramnia R et al (2018) Challenges and complication management in novel artificial Iris implantation. J Ophthalmol 2018:3262068

4. Spitzer MS, YoeruekE, Leitritz MA et al (2012) A new technique for treating posttraumatic aniridia with aphakia: first results of haptic fixation of a foldable intraocular lens on a foldable and custom-tailored iris prosthesis. Arch Ophthalmol 130:771-775

5. MayerCS, ReznicekL,HoffmannAE (2016) Pupillary reconstruction and outcome after artificial iris implantation. Ophthalmology 123:1011-1018

6. Yildirim TM, Khoramnia R, Masyk $M$ et al (2020) Aesthetics of iris reconstruction with a custom-made artificial iris prosthesis. PLoS ONE 15:e237616. https://doi.org/10.1371/journal. pone. 0237616

7. Baur ID, Mayer CS, Storr J, Khoramnia R (2021) Artificial-Iris-Implantation bei iatrogenem Irisdefekt nach Kataraktoperation [Artificial Iris Implantation in a Patient with latrogenic Iris Defect Following Cataract Surgery]. Klin Monbl Augenheilkd. https:// doi.org/10.1055/a-1353-5548. PMID: 33607679.

8. Mayer C, Tandogan T, Hoffmann AE et al (2017) Artificial iris implantation in various iris defects and lens conditions. J Cataract Refract Surg 43:724-731

9. Mayer CS, Baur I, Storr J et al (2020) Surgical management for silicone oil barrier of traumatic Aniridia with Aphakia: suturing of temporary 
Iris-diaphragm prior to final Iris-lens-diaphragm implantation. Clin Ophthalmol 14:4439

10. Mayer C, Baur ID, Storr J et al (2021) Complete anterior segment reconstruction: corneal transplantation and implantation of an iris prosthesis and $\mathrm{IOL}$ in a single surgery. Eur J Ophthalmol. https://doi.org/10.1177/1120672121991052

11. Mayer C, Khoramnia R (2021) “Double prosthesis implantation": biometry and refractive outcomes in combined intraocular lens and artificial iris surgery. Clin Ophthalmol 15:799-805. https://doi. org/10.2147/OPTH.S302224

12. Spitzer MS, Nessmann A, Wagner J et al (2016) Customized humanoptics silicone iris prosthesis in eyes with posttraumatic iris loss: outcomes and complications. Acta Ophthalmol 94:301-306

13. Mayer C, Hoffmann A (2015) Surgical treatment with an artificial iris. Ophthalmologe 112:865-868

14. Mayer CS, Laubichler AE, Masyk M et al (2019) Residual Iris retraction syndrome after artificial Iris implantation. Am J Ophthalmol 199:159-166

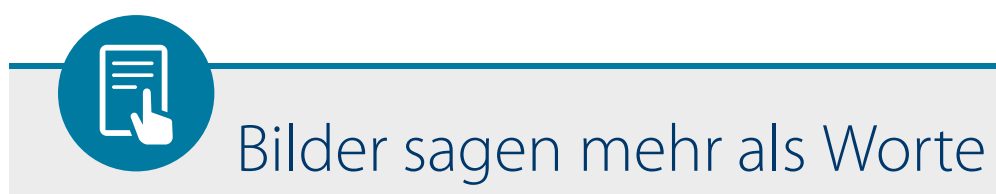

Wir suchen Ihre informativen und überraschenden Bilder!

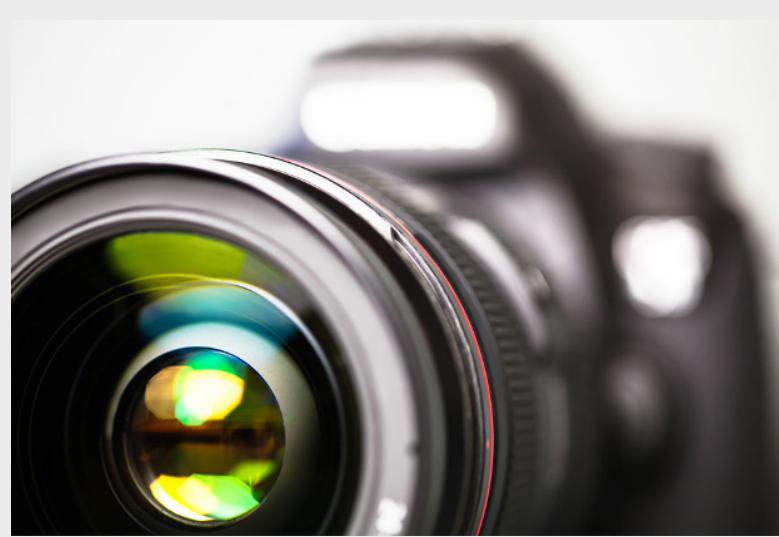

(c) Fotimmz / Fotolia

Verlag und Herausgebende von Der Ophthalmologe laden Sie ein, die aufschlussreichsten Bilder aus Ihrem Alltag mit der Community zu teilen.

Schicken Sie uns Ihre Aufnahme oder eine klinisch-pathologi- sche Korrelation mit einer prägnanten und aussagekräftigen Bildlegende.

Eine Auswahl der informativsten Schnappschüsse und klinisch-pathologischen Korrelationen werden dann, inklusive der Geschichte dahinter, in Der Ophthalmologe veröffentlicht. Wir freuen uns auf Ihre Beteiligung!

\section{Hinweise zur Einreichung:}

- 1 Abbildung bestehend aus max. sechs Einzelbildern (a-f); bei klinisch-pathologischer Korrelation unter Angabe von Färbung und Vergrößerung

- Aussagekräftiger Manuskript-Titel

- Bildlegende mit max. 2500 Zeichen inkl. Leerzeichen

- Max. 4 Autorinnen/Autoren sowie vollständige Korrespondenzadresse

Senden Sie Ihre Bilder an: Michal Meyer zu Tittingdorf Managing Editor von Der Ophthalmologe michal.meyerzutittingdorf@springer.com 